\title{
Advancing the Detection and Management of Diabetic Retinopathy with Ultra-widefield Retinal Imaging
}

\author{
David M Brown
}

Retina Consultants of Houston, Houston, Texas, US and The Methodist Hospital-Weill Cornell Medical College, New York, New York, US

$\mathrm{T}$ he incidence of diabetes in the US population has increased more than fourfold over the last several decades and a high proportion of these patients manifest diabetic eye disease, including diabetic retinopathy (DR) and diabetic macular edema (DME). Ultra-widefield (UWF) retinal imaging has emerged as a valuable tool in the evolving standard of care for DR, providing essential visualization of ischemia and related pathology across the retina, particularly in the periphery, where these signs may appear earliest but may not be detected by conventional fundus photography. Multimodal UWF imaging has helped correlate changes in the periphery with DR progression, providing important guidance for treatment planning and facilitating improved understanding of the underlying mechanisms of disease. Rapid capture, immediate retrieval and efficient sharing of UWF retinal images support a wide spectrum of care settings_including teleophthalmology programs - and facilitate patient education.

\section{Keywords}

Ultra-widefield retinal imaging, diabetic retinopathy, fluorescein angiography, retinal periphery, ischemia, pan-retinal photocoagulation, teleophthalmology

Disclosure: David M Brown is a consultant for Heidelberg, Optos, Optovue, and Zeiss.

Acknowledgements: Editorial assistance was provided by BioComm Network, Inc.

Compliance with Ethics: This study involves a review of the literature and did not involve any studies with human or animal subjects performed by any of the authors.

Authorship: The named author meets the International Committee of Medical Journal Editors (ICMJE) criteria for authorship of this manuscript, takes responsibility for the integrity of the work as a whole, and has given final approval to the version to be published.

Open Access: This article is published under the Creative Commons Attribution Noncommercial License, which permits any noncommercial use, distribution, adaptation, and reproduction provided the original author(s) and source are given appropriate credit.

Received: January 27, 2017

Accepted: March 10, 2017

Citation: US Ophthalmic Review, 2017;10(1):23-6

Corresponding Author: David M Brown,

Retina Consultants of Houston, 6560 Fannin,

Suite 750 Houston, TX 77030, US.

E: dmbmd@houstonretina.com

Support: The publication of this article was supported by Optos. The views and opinions expressed are those of the author and do not necessarily reflect those of Optos.
Diabetic retinopathy (DR) is one of the leading causes of adult vision loss in the developed world. ${ }^{1,2}$ Epidemiological and demographic factors, including the rising rates of diabetes related to obesity and an aging population, are driving the incidence of diabetic eye disease inexorably higher. Over the past 35 years, the number of Americans diagnosed with diabetes increased fourfold, from 5.5 million in 1980 to 22.0 million in 2014. ${ }^{3}$ In 2014, approximately 8 million Americans with diabetes were undiagnosed, making the total incidence of the disease in the US more than 29 million, or $9.3 \%$ percent of the population. ${ }^{4}$ Analyses based on data collected a decade ago in the National Health and Nutrition Examination Survey (NHANES) suggest that nearly a third of all adults with diabetes in the US had signs of DR. This rate was even higher among specific populations of diabetic patients, with more than a third of Mexican Americans and nearly $40 \%$ of African Americans having diabetic eye disease. Patients in these groups were also more likely to present with severe disease and vision loss. Overall, an estimated 4.2 million adults had DR at that time and 655,000 had disease severe enough to be vision threatening. ${ }^{5}$ Given the data showing that the at-risk population has continued to grow at a similar rate, the clinical community must take aggressive action toward earlier identification and treatment of patients with DR. Meeting this challenge requires improved access to screening and referral as well as enhanced strategies for earlier detection, evaluation, treatment, and assessment of treatment success.

In this setting, digital, multi-modality ultra-widefield (UWFTM) retinal imaging (Optos, plc., Dunfermline, Scotland) provides a technological cornerstone for the evolving standard of care. With a single, non-contact capture, UWF retinal imaging allows high-resolution visualization of up to $82 \%$, or 200 degrees, of the retina (producing the digital optomap ${ }^{\circledR}$ ), displaying the most retinal surface area of any available imaging system. ${ }^{6}$ Optos systems incorporate a proprietary scanning laser ophthalmoscope (SLO) and ellipsoidal mirror, engineered specifically for UWF imaging. The SLO utilizes two low-power lasers that scan the retina simultaneously, with the green 'red free' wavelength (532 nm) permitting visualization of the sensory retina to the retinal pigment epithelium (RPE) and the red wavelength $(635 \mathrm{~nm})$ reaching deeper structures from the RPE to the choroid. The latest generation Optos UWF platform, California, utilizes enhanced optical hardware and software to improve resolution during scanning, producing clear, high-resolution images of virtually the entire retina. The automatic montage feature increases the field of view in many eyes to greater than $95 \%$. California provides multi-modality imaging options including color, autofluorescence (AF), fluorescein angiography (FA), and indocyanine green angiography (ICG). 
Figure 1: optomap of proliferative diabetic retinopathy with ETDRS 7-standard field overlay

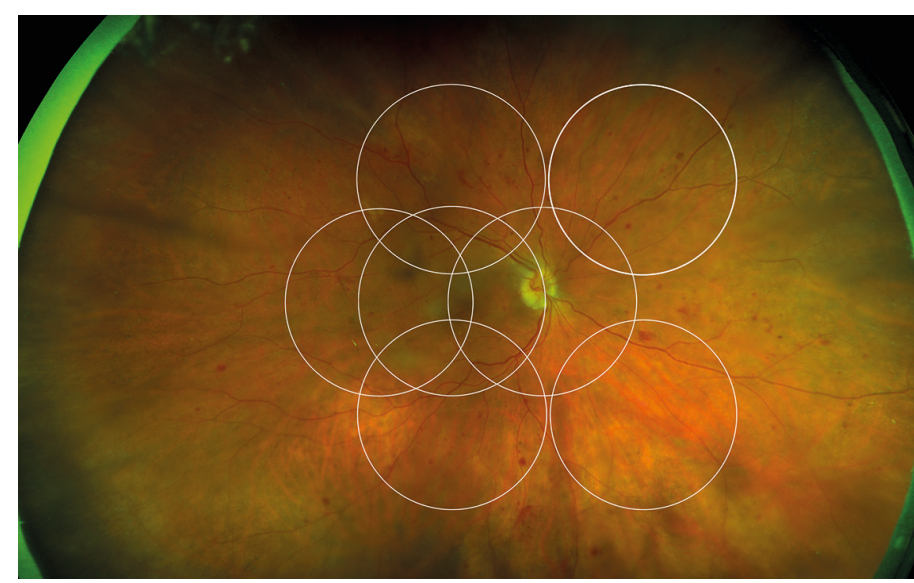

ETDRS = Early Treatment Diabetic Retinopathy Study.

Figure 2: optomap demonstrating proliferative diabetic retinopathy

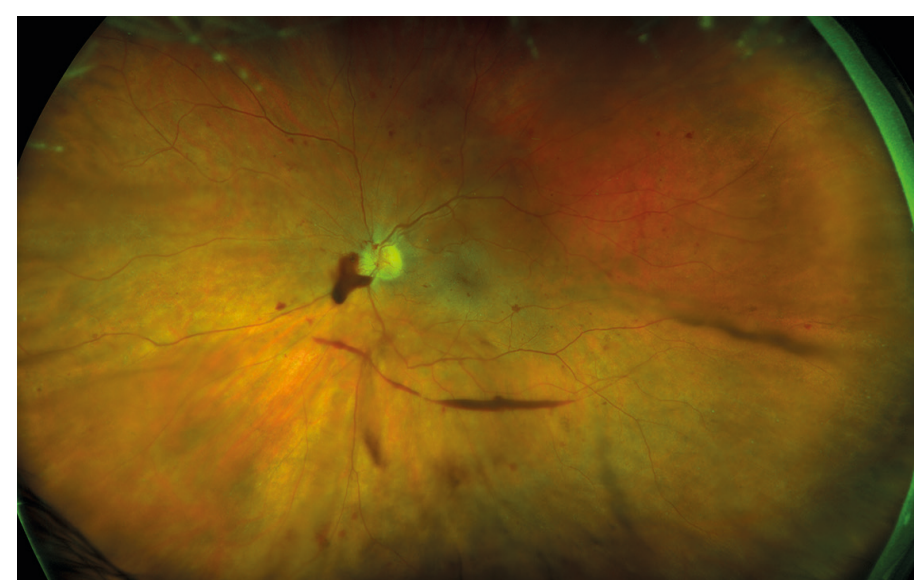

Figure 3: optomap FA demonstrating proliferative diabetic retinopathy with areas of non-perfusion and leakage outside the central pole

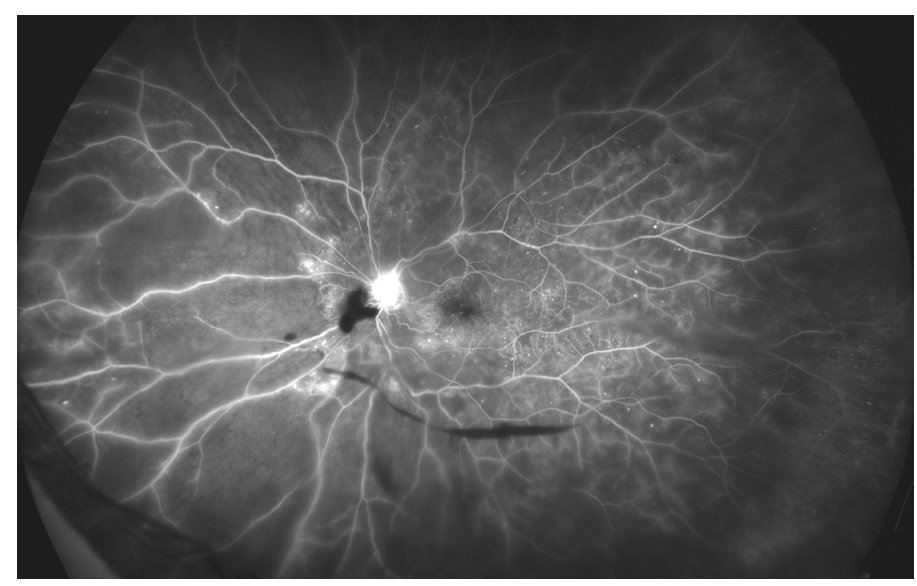

$F A=$ fluorescein angiography. Image courtesy of Srinivas Sadda, MD

\section{Ultra-widefild imaging in diabetic retinopathy detection and evaluation}

The potential role of UWF imaging in the detection of DR has been described for nearly a decade. ${ }^{8}$ Early studies established the excellent correspondence between conventional Early Treatment Diabetic Retinopathy Study (ETDRS) 7-fields and optomap in evaluating DR and verified that UWF enabled the visualization of a much larger area of the peripheral retina (see Figure 1). 9.10 One comparison in 103 patients with diabetes confirmed the correlation of conventional fundus photography and UWF images in DR assessment, while demonstrating that optomap required less than half the image capture time.11 Because at least two-thirds of potential retinal pathology lies in the periphery, UWF color and FA images allow the detection of vascular nonperfusion, peripheral microaneurysms, neovascularization, and vascular leakage that are missed by conventional ETDRS 7-fields photography. These peripheral lesions often occur earlier in the disease course; they may also provide evidence of more advanced disease. ${ }^{12-14}$ Combining UWF capabilities with ocular coherence tomography (OCT) has also been shown to have clinical utility in the assessment of DR. ${ }^{15}$

The capabilities of UWF retinal imaging are proving particularly useful in efforts to expand screening programs for DR, particularly in teleophthalmology settings for underserved, high-risk populations. Because optomap images can be captured, stored, and shared easily, even between practices that do not have Optos imaging systems, diagnostic efficiency, referral speed, and resource utilization can be improved in an increasingly time- and cost-constrained environment. In one diabetes teleophthalmology program, utilization of nonmydriatic UWF imaging shortened the time required for image evaluation by $28 \%$ and reduced the ungradable rate to less than $3 \%$, compared with conventional imaging, while increasing the detection of DR by $17 \%{ }^{16}$ The same group studied nonmydriatic UWF imaging in a multi-state DR ocular telehealth program administered for the Indian Health Service. They found that, compared with non-mydriatic fundus photography, UWF imaging in this setting reduced the ungradable image rate by $81 \%$, increased DR detection by nearly twofold, and identified peripheral lesions suggestive of more severe DR in nearly $10 \%$ of patients. ${ }^{17}$

\section{The importance of examining the periphery in diabetic retinopathy}

In addition to the fact that a significant amount of pathology associated with DR lies outside the central fields, a growing body of evidence points to the retinal periphery as a locus of early signs of diabetic eye disease and a source of important indications of its progression. Ischemia, a key driver of progression in DR, may appear first in the retinal periphery, well beyond the range of traditional angiography. A close association between predominantly peripheral lesions, the extent of nonperfusion and ischemia, and DR severity has been demonstrated with UWF FA. ${ }^{18}$ Analogous to trees shedding peripheral leaves in times of drought, and frostbite affecting distal extremities as the body works to preserve critical central organs, the human retina can experience marked peripheral capillary loss well before evidence of central pathology. Identifying the presence and extent of ischemia across the entire retina may permit more accurate assessment and effective management, particularly in staging anti-vascular endothelial growth factor (VEGF) treatments with photocoagulation (see Figures 2 and 3). ${ }^{19}$ Indeed, the value of UWF imaging in linking the extent of peripheral ischemia to the presence or potential development of diabetic macular edema (DME) and worsening disease, has been evaluated in several studies. Peripheral 
ischemia, detected on UWF FA, has been strongly correlated with DME in treatment-naïve patients. ${ }^{20}$ Other investigators have proposed a new ischemic index based on UWF findings. This work established the relationship between peripheral nonperfusion, severity of DR, and recalcitrant macular edema, while suggesting that areas of untreated peripheral nonperfusion may be the source of mediators of ischemia and disease progression. ${ }^{21}$ The potential prognostic value of peripheral lesions identified with UWF was confirmed in a study that demonstrated the association of these findings with a significantly increased risk of disease progression over a fouryear period, independent of baseline severity of DR or diabetic control. ${ }^{22}$ These observations suggest that the established ' $4-2-1$ ' rule for identifying severe non-proliferative DR and a high risk for disease progression, based on the evaluation of signs identified through conventional imaging of the central fields (diffuse intraretinal hemorrhages and microaneurysms in four quadrants, venous beading in two or more quadrants, or intraretinal microvascular abnormalities in one or more quadrants), may underestimate DR severity compared with findings in the periphery from UWF imaging. A multi-year study that will enroll at least 350 participants is being conducted by the Diabetic Retinopathy Clinical Research Network (DRCRnet) to further establish the impact of UWF evaluation of the retina on the assessment of DR and in predicting rates of DR progression over time, compared with conventional ETDRS 7-fields. ${ }^{23}$

\section{The use of ultra-widefield retinal imaging in treatment planning}

Visualization of the entire retina may provide a rationale to initiate treatment earlier and arrest progression sooner. This hypothesis is supported by the results of the Ranibizumab Injection in Subjects With Clinically Significant Macular Edema With Center Involvement Secondary to Diabetes Mellitus (RIDE and RISE) studies, which demonstrated that greater improvements in vision were achieved in eyes treated within the first two years, compared to eyes with chronic DME associated with irreversible damage to the retina. ${ }^{24}$ Findings from peripheral examination have allowed us to intervene in cases of proliferative retinal vein occlusion before a hemorrhage occurs, reduce the number of vitrectomies we perform for floaters, and identify neovascularization earlier. The value of acting earlier is supported by data confirming that patients with post vitrectomy diabetic vitreous hemorrhage display higher rates of neovascularization, nonperfusion, and late vascular leakage in the periphery. ${ }^{25}$

Peripheral findings also provide important information about which areas of the retina should be treated. Our experience suggests that identifying areas of ischemia with UWF allows more precise targeting of pan-retinal photocoagulation (PRP) to retinal pathology while sparing areas that remain adequately perfused. Sparing of useful visual field (especially nasal and superior retina) can help preserve inferior and temporal depth perception important for walking down stairs or curbs and making driving safer. Finally, UWF retinal imaging can be employed to evaluate treatment success (see Figures 4, 5, and 6). For example, documentation with UWF FA of reperfusion in areas of nonperfusion following anti-VEGF treatment was recently described. ${ }^{26}$

\section{Improving clinical and patient communication}

The immediate availability of multimodal optomap images following singleshot, nonmydriatic capture permits more efficient evaluation at the point of care. Remote review at a reading center or in other practices is facilitated by browser-based, platform-independent (DICOM compatible), Health
Figure 4: optomap demonstrating diabetic retinopathy with panretinal photocoagulation and pre-retinal and retinal hemorrhages in the periphery

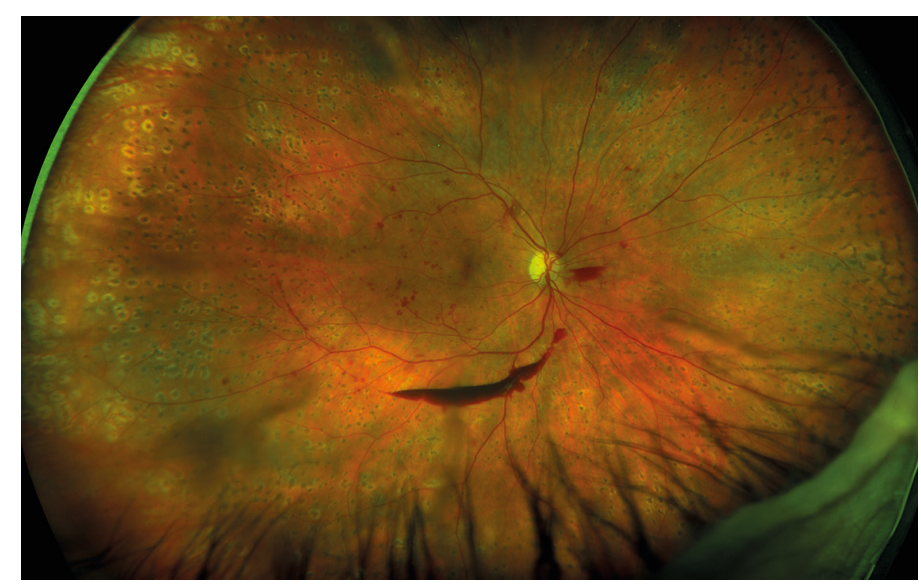

Image courtesy of Paul Stanga, MD.

Figure 5: optomap of diabetic retinopathy with panretinal photocoagulation in the periphery

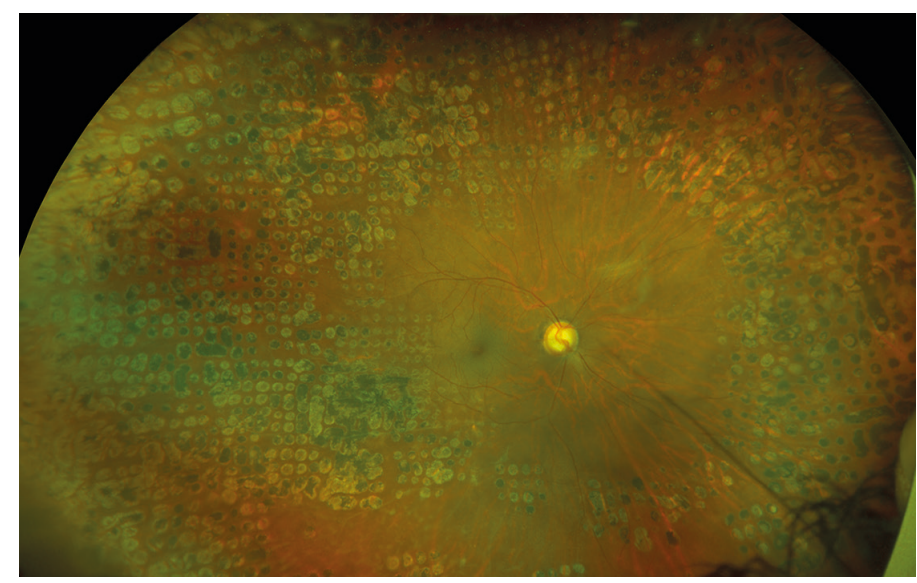

Image courtesy of Srinivas Sadda, MD.

Figure 6: optomap FA of diabetic retinopathy with panretinal photocoagulation in the periphery with a quiet central pole

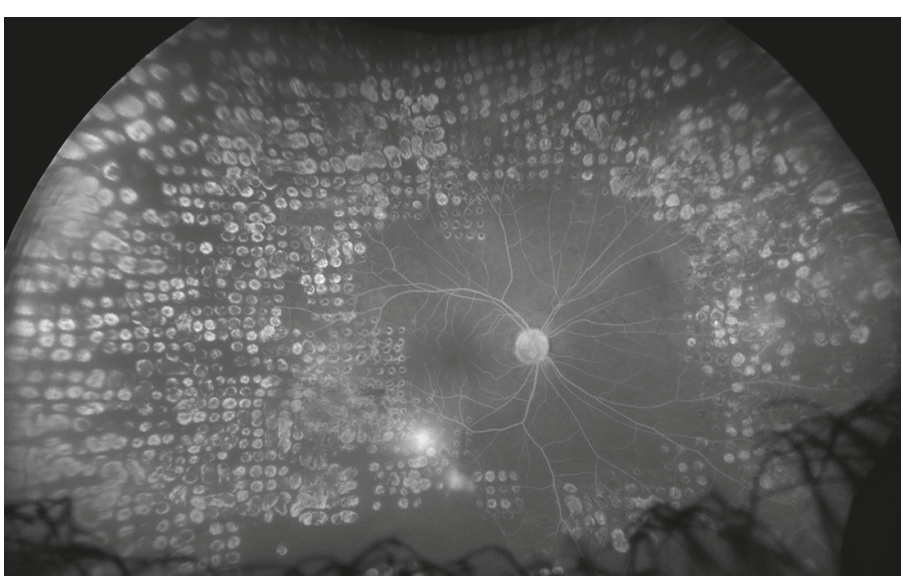

$F A=$ fluorescein angiography. Image courtesy of Srinivas Sadda, MD. 
Insurance Portability and Accountability Act (HIPAA)-compliant image sharing and cloud storage. This ease of image sharing is particularly useful in referral situations or teleophthalmology settings.

In addition to efficient evaluation and annotation by the clinical team, a single optomap or historical comparisons can be reviewed with the patient without delay during the office visit on a computer screen or tablet. While diabetes affects small blood vessels all over the body, DR is often the first manifestation of end-organ disease that can be detected. Many patients with diabetes are non-compliant with blood sugar control and are in denial that this is detrimental to their physical health and end organs. We have found that counseling a patient on improving blood sugar control while demonstrating with UWF imaging that damage is already occurring in their peripheral retina is often a very effective and convincing approach to education and behavior modification. We review the optomap with the patient and their care partners, pointing out microaneuryms and capillary non-perfusion. We advise them that if damage is occurring in the retina, then it is also happening in the heart, the kidneys, the brain, and throughout their body. In our experience, the immediacy of this vivid demonstration of the impact of their lack of adherence on the blood vessels in their body, particularly if historical comparison has indicated progression, helps patients and their loved ones realize that they need to become more serious about glycemic control and other aspects of their treatment plan.

\section{Conclusion}

The dramatic increase in the incidence of diabetes and associated eye disease will continue to pose a challenge for the clinical community. In this environment, UWF retinal imaging is valuable for effective screening, refinement of treatment decision-making, and advancement of our understanding of the disease process itself. By facilitating earlier diagnosis, earlier treatment, and more effective patient education, UWF retinal imaging is helping to shape the new standard of care for DR that will preserve vision for more of our patients with diabetes.
1. Bourne RRA, Stevens GA, White RA, et al., Causes of vision loss worldwide, 1990-2010: a systematic analysis, Lancet, 2013:1:e339-e349.

2. Lee R, Wong TY, Sabanayagam C, Epidemiology of diabetic retinopathy, diabetic macular edema and related vision loss, Eye Vis, 2015;2:17

3. Centers for Disease Control and Prevention, National Center for Health Statistics, Division of Health Interview Statistics, data from the National Health Interview Survey. Available at: www.cdc.gov/ diabetes/statistics/prev/national/figpersons.htm (accessed March 13, 2017)

4. Centers for Disease Control and Prevention, National Diabetes Statistics Report: Estimates of Diabetes and Its Burden in the United States, 2014, Atlanta, GA: US, Department of Health and Human Services, 2014

5. Zhang X, Saaddine JB, Chou C, et al., Prevalence of diabetic retinopathy in the United States, 2005-2008, JAMA 2010;304:649-56.

6. Witmer MT, Parlitsis G, Patel S, et al., Comparison of ultra-widefield fluoresceuin angiography with the Heidelberg Spectralis ${ }^{\circledR}$ noncontact ultra-widefield module versus the Optos ${ }^{\circledast}$ Optomap ${ }^{\circledR}$ Clin Ophthalmol, 2013;7:389-94.

7. Optos. Data on file. 2017

8. Kaines A, Oliver S, Reddy S, Schwartz SD, Ultrawide angle angiography for the detection and management of diabetic angiography for the detection and managemento

9. Kernt M, Hadi I Pinter F et al., Assessment of diabetic retinopathy using nonmydriatic ultra-widefield scanning laser ophthalmoscopy (optomap) compared with ETDRS 7-field stereo photography, Diabetes Care, 2012;35:2459-63.

10. Kernt M, Pinter F, Hadi I, et al., Diabetic retinopathy: comparison of the diagnostic features of ultra-widefield scanning laser ophthalmoscopy Optomap with ETDRS 7-field fundus photography [article in German], Ophthalmologe, 2011:108:117-23.

11. Silva PS, Cavallerano JD, Sun JK, et al., Nonmydriatic ultrawide field retinal imaging compared with dilated standard 7 -field 35-mm photography and retinal specialist examination for evaluation of diabetic retinopathy, Am J Ophthalmol, 2012;154:549-59.

12. Wessel MM, Aaker GD, Parlitsis $G$ et al., Ultra-wide-field angiography improves the detection and classification of diabetic retinopathy, Retina, 2012;32:785-91.

13. Kong M, Lee MY, Ham DI, Ultrawide-field fluorescein angiography for evaluation of diabetic retinopathy, Korean J Ophthalmol, 2012;26:428-31.

14. Silva PS, Cavallerano JD, Sun JK, et al., Peripheral lesions identified by mydriatic ultrawide field imaging: distribution and potential impact on diabetic retinopathy severity, Ophthalmology potential impact on

15. Manjunath $V$, Papastavrou V, Steel DHW, et al., Wide-field imaging and OCT vs clinical evaluation of patients referred from diabetic retinopathy screening, Eye, 2015;29:416-23.

16. Silva PS, Cavallerano JD, Tolls $D$, et al., Potential efficiency benefits of nonmydriatic ultrawide field retinal imaging in an ocular telehealth diabetic retinopathy program, Diabetes Care, 2014;37:50-5.

17. Silva PS, Horton MB, Clary D, et al., Identification of diabetic retinopathy and ungradable image rate with ultrawide field imaging in a national teleophthalmology program, Ophthalmology , 2016;123:1360-7

18. Silva PS, Dela Cruz AJ, Ledesma MG, et al., Diabetic retinopathy severity and peripheral lesions are associated with nonperfusion on ultrawide field angiography, Ophthalmology, 2015;122:2465-72.

19. Takamura Y, Tomomatsu T, Matsumura T, et al., The effect of photocoagulation in ischemic areas to prevent recurrence of diabetic macular edema after intravitreal bevacizumab injection, Invest Ophthalmol Vis Sci, 2014; 55:4741-6.

20. Wessel MM, Nair N, Aaker GD, et al., Peripheral retinal ischaemia, as evaluated by ultra-widefield fluorescein angiography, is associated with diabetic macular oedema, Br $j$ Ophthalmol, 2012:96:694-8.

21. Patel RD, Messner LV, Teitelbaum B, et al., Characterization of ischemic index using ultra-widefield fluorescein angiography in patients with focal and diffuse recalcitrant diabetic macular edema, Am J Ophthalmol, 2013;155:1038-44.

22. Silva PS, Cavallerano JD, Haddad NMN, et al., Peripheral lesions identified on ultrawide field imaging predict increased risk of diabetic retinopathy progression over 4 years, Ophthalmology, 2015:122:949-56.

23. DRCR.net Protocol. Available at: http://drcrnet.jaeb.org/Studies aspx?RecID=239 (accessed March 31, 2017).

24. Brown DM, Nguyen QD, Marcus DM, et al., Long-term outcomes of ranibizumab therapy for diabetic macular edema: The 36-month results from two phase III trials (RISE and RIDE), Ophthalmology, 2013;120:2013-22.

25. Kim DY, Kim J-G, Kim JY, et al., Ultra-widefield fluorescein angiographic findings in patients with recurrent vitreous hemorrhage after diabetic vitrectomy, Invest Ophthalmol Vis Sci, 2014;55:7040-6.

26. Levin AM, Rusu I, Orlin A, et al., Retinal reperfusion in diabetic retinopathy following treatment with anti-VEGF intravitreal injections, Clinical Ophthalmology, 2017:11:193-200. 